\title{
Editorial: Lupus and the Brain: Advances in Neuropsychiatric Systemic Lupus Erythematosus
}

\author{
Antonis Fanouriakis $^{1 *}$, George Bertsias ${ }^{2}$ and Marcello Govoni ${ }^{3}$ \\ 'Rheumatology and Clinical Immunology Unit, "Attikon" University Hospital, Athens, Greece, ${ }^{2}$ Rheumatology, Clinical \\ Immunology and Allergy, University Hospital of Heraklion, Heraklion, Greece, ${ }^{3}$ Section of Rheumatology, Department of \\ Medical Sciences, University of Ferrara, Azienda Ospedaliero-Universitaria Sant'Anna Ferrara, Ferrara, Italy
}

Keywords: systemic lupus erythematosus, central nervous system, attribution, diagnosis, biomarkers

\section{Editorial on the Research Topic}

\section{Lupus and the Brain: Advances in Neuropsychiatric Systemic Lupus Erythematosus}

\section{OPEN ACCESS}

Edited by:

João Eurico Fonseca,

Universidade de Lisboa, Portugal

Reviewed by:

Guo-Min Deng,

Huazhong University of Science and

Technology, China

Cheng-De Yang,

Shanghai Jiao Tong University, China

*Correspondence:

Antonis Fanouriakis

afanour@med.uoa.gr

Specialty section:

This article was submitted to

Rheumatology,

a section of the journal

Frontiers in Medicine

Received: 20 February 2019 Accepted: 04 March 2019

Published: 26 March 2019

Citation:

Fanouriakis A, Bertsias $G$ and Govoni M (2019) Editorial: Lupus and

the Brain: Advances in

Neuropsychiatric Systemic Lupus Erythematosus. Front. Med. 6:52.

doi: 10.3389/fmed.2019.00052
As systemic lupus erythematosus (SLE) is a notoriously demanding disease, involvement of the nervous system, collectively termed neuropsychiatric SLE (NPSLE), represents the foremost diagnostic and therapeutic challenge for the treating physician. Reasons for this include, but are not limited to, the wide heterogeneity of its presentation, the lack of specific markers and a diagnostic "gold-standard," as well as the paucity of well-designed clinical trials to guide therapy (1). In this Research Topic for the Frontiers in Medicine, four articles by distinguished contributors touch upon different aspects of NPSLE, in an attempt to elucidate the optimal approach to this challenging group of patients.

In 1999, the American College of Rheumatology (ACR) research committee published a set of case definitions for 19 NPSLE syndromes, in an effort to homogenize terminology for research and clinical practice purposes. These case definitions involve both the central and the peripheral nervous system, are categorized into focal and diffuse and have a wide heterogeneity that ranges from overt manifestations such as stroke, seizures and psychosis, to headache or subtle abnormalities of cognitive function (2). An "outsider" of the NPSLE field would rightfully wonder straight away: How can a patient with headache, a patient with psychosis and a patient with peripheral neuropathy, three very different conditions, be grouped under the same "umbrella" term (which implies common pathophysiology and possibly treatment)? Are these patients the same, or should they be approached in the same way?

The now considered "classic" population-based study by Ainiala et al., published shortly after the ACR NPSLE definitions, questioned the value of including the so-called "common" or "minor" manifestations (including headache, anxiety disorder, mild mood disorder and cognitive impairment, and polyneuropathy without electrophysiologic confirmation) in the spectrum of NPSLE (3). By excluding these manifestations, specificity for the diagnosis of "frank" NPSLE increased from 46 to $93 \%$, since the former are very common also in the general population. This, and other observations, have steered the discussions as to what actually constitutes "NPSLE." The actual existence of "lupus headache," as a distinct NPSLE syndrome, has been questioned by some authors $(4,5)$. Bearing these in mind, in this Research Topic, Vivaldo et al. critically revisit the original ACR nomenclature to comment on its advantages, but also potential pitfalls. While the authors acknowledge the major step forward accomplished by the ACR nomenclature to homogenize NPSLE research, they suggest that a more comprehensive model, taking into account also the issue of attribution, could move the field forward, to optimize patient care. 
The issue of attribution represents an important unmet need in NPSLE (6). In a lupus patient with a given neuropsychiatric manifestation, correctly attributing the latter to the disease per se or not has important therapeutic implications. Given that in NPSLE "nothing is specific," various attempts have been made to construct sets of rules and attribution models. In a relevant review, Bortoluzzi et al. present an overview of previously proposed "hints" for attribution, focusing on their own formidable effort, which led to the attribution algorithm of the Italian Study Group for NPSLE (7). The novelty of the Italian model was the inclusion of "favoring" factors, i.e., factors whose presence is supportive of diagnosing primary NPSLE. These include the presence of generalized disease activity, abnormal brain imaging and cerebrospinal fluid (CSF) analysis, aPL positivity and others. This algorithm has now been independently validated and may provide significant help to physicians and center with less expertise in SLE; it also offers, to researchers in the field, a valuable tool for a more rigorous and standardized selection of NPSLE patients for epidemiological studies and clinical trials (8). Nevertheless, to emphasize the indispensable role of multidisciplinary and longitudinal judgment in NPSLE, a subsequent study testing the proposed models found that, upon re-evaluation after 3-8 months from initial attribution, almost $15 \%$ of patients initially classified as primary NPSLE were deemed as having neuropsychiatric events not attributed to SLE (9).

Irrespective of the clinical syndrome, the pathophysiology of NPSLE is traditionally considered a combination of autoantibody-mediated neuronal or vascular injury, intrathecal production of inflammatory cytokines, disruption of the bloodbrain barrier, and accelerated atherosclerosis (1). A certain degree of cerebral vasculopathy is evident in NPSLE by the common finding of white matter T2-hyperintense lesions in conventional brain MRI of patients with NPSLE, but also from multiple studies using novel brain imaging techniques, which show hypoperfusion even in normal appearing white matter (10-13). Teixeira and Tam have performed a systematic literature review, to provide modern insights on the pathophysiology of premature

\section{REFERENCES}

1. Schwartz N, Stock AD, Putterman C. Neuropsychiatric lupus: new mechanistic insights and future treatment directions. Nat Rev Rheumatol. (2019) 15:137-52. doi: 10.1038/s41584-018-0156-8

2. The American College of Rheumatology nomenclature and case definitions for neuropsychiatric lupus syndromes. Arthritis Rheum. (1999) 42:599-608.

3. Ainiala H, Hietaharju A, Loukkola J, Peltola J, Korpela M, Metsanoja R, et al. Validity of the new american college of rheumatology criteria for neuropsychiatric lupus syndromes: a population-based evaluation. Arthritis Rheum. (2001) 45:419-23. doi: 10.1002/1529-0131(200110)45:5<419::AID-ART360>3.0.CO;2-X

4. Mitsikostas DD, Sfikakis PP, Goadsby PJ. A meta-analysis for headache in systemic lupus erythematosus: the evidence and the myth. Brain. (2004) 127(Pt 5):1200-9. doi: 10.1093/brain/ awh146

5. Hanly JG, Urowitz MB, O'Keeffe AG, Gordon C, Bae SC, Sanchez-Guerrero J, et al. Headache in systemic lupus erythematosus: results from a prospective, international inception cohort study. Arthritis Rheum. (2013) 65:2887-97. doi: 10.1002/art.38106 and accelerated atherosclerosis in SLE. Importantly, the authors provide a thorough overview of molecular biomarkers that have been tested in relation to cardiovascular/cerebrovascular events and mortality; they also outline the various imaging surrogate markers for atherosclerosis, including flow-mediated dilatation of the brachial artery, carotid intima media thickness and pulse wave velocity. Albeit these studies have added valuable information, no single surrogate marker (molecular or imaging) has yet shown a clear association with "hard" endpoints, i.e., vascular events or cardiovascular mortality.

Along the same lines, in a Perspective article, Magro-Checa et al. present the current state-of-the-art regarding the utility of biomarkers (laboratory and neuroimaging) for prediction and diagnosis of NPSLE, in particular. The authors give a comprehensive review of serum and CSF biomarkers; notably, from the bulk of experimental biomarkers, only serum lupus anticoagulant and CSF interleukin-6 have reached clinical usefulness. Following also an extensive description of the various novel brain imaging techniques which have been utilized in NPSLE, Magro-Checa et al. end with attractive proposals to improve the scant performance of the pursuit for biomarkers in NPSLE, ranging from definition of NPSLE and better characterization of patient groups, to the combination of laboratory and multimodal neuroimaging markers.

In conclusion, our understanding of NPSLE has witnessed significant advances over the last decades. Nevertheless, to optimize patient care, future research should focus on a better characterization of patient cohorts, through multicentre international collaborations, as well as establishment of universally accepted guidelines for diagnosis, evaluation of disease activity, identification of more specific outcome measures and assessment of response to treatment.

\section{AUTHOR CONTRIBUTIONS}

All authors listed have made a substantial, direct and intellectual contribution to the work, and approved it for publication.

6. Tay SH, Mak A. Diagnosing and attributing neuropsychiatric events to systemic lupus erythematosus: time to untie the Gordian knot? Rheumatology. (2017) 56(suppl_1):i14-i23. doi: 10.1093/rheumatology/ kew338

7. Bortoluzzi A, Scire CA, Bombardieri S, Caniatti L, Conti F, De Vita S, et al. Development and validation of a new algorithm for attribution of neuropsychiatric events in systemic lupus erythematosus. Rheumatology. (2015) 54:891-8. doi: 10.1093/rheumatology/keu384

8. Fanouriakis A, Pamfil C, Rednic S, Sidiropoulos P, Bertsias G, Boumpas DT. Is it primary neuropsychiatric systemic lupus erythematosus? Performance of existing attribution models using physician judgment as the gold standard. Clin Exp Rheumatol. (2016) 34:910-7.

9. Magro-Checa C, Zirkzee EJ, Beaart-van de Voorde LJJ, Middelkoop $\mathrm{HA}$, van der Wee NJ, Huisman MV, et al. Value of multidisciplinary reassessment in attribution of neuropsychiatric events to systemic lupus erythematosus: prospective data from the Leiden NPSLE cohort. Rheumatology. (2017) 56:1676-83. doi: 10.1093/rheumatology/ kex019

10. Luyendijk J, Steens SC, Ouwendijk WJ, Steup-Beekman GM, Bollen EL, van der Grond J, et al. Neuropsychiatric systemic lupus erythematosus: lessons 
learned from magnetic resonance imaging. Arthritis Rheum. (2011) 63:72232. doi: 10.1002/art.30157

11. Magro-Checa C, Ercan E, Wolterbeek R, Emmer B, van der Wee NJ, Middelkoop HA, et al. Changes in white matter microstructure suggest an inflammatory origin of neuropsychiatric systemic lupus erythematosus. Arthritis Rheumatol. (2016) 68:1945-54. doi: 10.1002/art. 39653

12. Papadaki E, Fanouriakis A, Kavroulakis E, Karageorgou D, Sidiropoulos P, Bertsias G, et al. Neuropsychiatric lupus or not? Cerebral hypoperfusion by perfusion-weighted MRI in normal-appearing white matter in primary neuropsychiatric lupus erythematosus. Ann Rheum Dis. (2018) 77:441-8. doi: 10.1136/annrheumdis-2017-212285

13. Ramage AE, Fox PT, Brey RL, Narayana S, Cykowski MD, Naqibuddin $\mathrm{M}$, et al. Neuroimaging evidence of white matter inflammation in newly diagnosed systemic lupus erythematosus. Arthritis Rheum. (2011) 63:3048-57. doi: $10.1002 /$ art.30458

Conflict of Interest Statement: The authors declare that the research was conducted in the absence of any commercial or financial relationships that could be construed as a potential conflict of interest.

Copyright (c) 2019 Fanouriakis, Bertsias and Govoni. This is an open-access article distributed under the terms of the Creative Commons Attribution License (CC BY).

The use, distribution or reproduction in other forums is permitted, provided the original author(s) and the copyright owner(s) are credited and that the original publication in this journal is cited, in accordance with accepted academic practice. No use, distribution or reproduction is permitted which does not comply with these terms. 